EPJ Web of Conferences 41, 11004 (2013)

DOI: $10.1051 /$ epjconf/20134111004

C) Owned by the authors, published by EDP Sciences, 2013

\title{
Two-dimensional spectroscopy using dual acousto-optic pulse shapers for complete polarization, phase and amplitude control
}

\author{
P. Tyagi ${ }^{1}$, J. I. Saari ${ }^{1}$, V. Crozatier ${ }^{2}$, N. Forget ${ }^{2}$, P. Kambhampati ${ }^{1, *}$ \\ ${ }^{1}$ Department of Chemistry, McGill University, Montreal, Quebec H3A 0B8, Canada \\ ${ }^{2}$ FASTLITE, Centre scientifique d'Orsay - Bât.503, Plateau du Moulon - BP 45, Orsay, France
}

\begin{abstract}
We demonstrate a pulse-shaper capable of independent polarization, phase and amplitude control over each pulse. The set-up is compact and easily switchable between pump-probe and collinear geometries.
\end{abstract}

\section{Introduction}

2D spectroscopy has emerged as a powerful tool that can determine the complete nonlinear optical response of a system up to third-order. By providing access to specific quantum mechanical pathways, it allows for the measurement of vibrational and electronic couplings, energy transfer and multiexciton correlations, among other observables. 2D experiments can be implemented in different beam geometries, each having its advantages. For example, the boxcar geometry offers backgroundfree detection, thereby eliminating the need for phase cycling [1]. The collinear geometry, desirable for its simplicity, is easy to extend to higher-order experiments, and allows for both fluorescence and transmission detected experiments [2]. The pump-probe geometry has the advantage of directly measuring perfectly phased 2D spectra as it emits both rephasing and non-rephasing components in the same direction $[3,4]$.

Traditionally, the pump-probe configuration employs a single pulse shaper to generate a phasecoherent pump pulse pair, and the probe pulse, which also acts as the local oscillator, is derived from a second source such as a white light continuum or a non-collinear OPA [4,5]. Unfortunately, this arrangement does not provide phase stability between the pump and the probe pulses and hence, cannot be used for two-quantum (2Q) measurements. Also, the pulse shaper cannot independently control the polarization of the pump pulses, which is essential for the study of optical selection rules in systems such as quantum dots and quantum wells.

We overcome these limitations by using dual pulse shapers (Acousto-Optic Programmable Dispersive Filters (AOPDFs): WR25, low-jitter operation), which enable independent polarization, phase and amplitude control over each pulse. In addition to being extremely compact, this set-up is easily switchable between pump-probe and collinear geometries, allowing for detection in both phase-cycling and phase-matching arrangements (Fig.1a-b). Fast update rates of the AOPDF enable single-shot measurements at $1 \mathrm{kHz}$, significantly reducing the data acquisition time. Also, we precompress the unshaped pulse before the shaping set-up using GRISMs (combination of gratings and prisms); this compensates for part of the dispersion of the AOPDFs and allows us to produce a maximum delay of $>4$ ps between the shaped pulses. 


\section{Experiment}

The set-up uses two AOPDFs to generate phase and amplitude shaped pulses, which are combined using a polarizing cube (PC2) to produce the desired polarization state (Fig.1a). The translation of PC2 using a delay stage controls the spatial separation between the pulses from the two AOPDFs and allows for easy switching between pump-probe and collinear geometries (Fig.1b). The phase between the two pulses is measured using spectral interferometry, and the polarization is measured using a simplified Mueller ellipsometer shown in Fig. 2a. The ellipsometer measures two quadratures of the polarization state in both linear and circular polarization bases, thus characterizing the polarization of the shaped pulses.

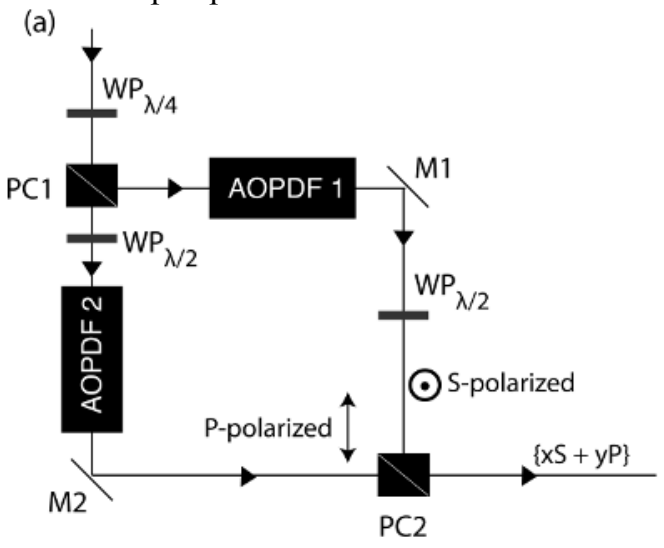

(b)

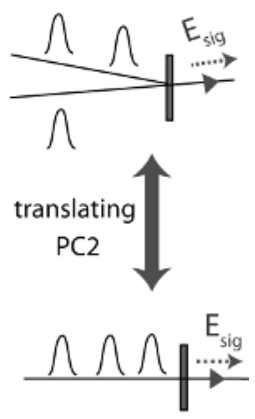

Fig. 1. (a) The broadband output of a non-collinear OPA is separated into orthogonal polarization components using a polarizing cube (PC1). Each component is sent through an AOPDF to produce shaped pulses, which are then combined using PC2 to produce desired polarization. (b) Translating PC2 spatially separates the shaped pulses from the two AOPDFs, making it easy to switch between the pump-probe (top) and collinear (bottom) geometries.

\section{Results and Discussion}

The synchronization of the two AOPDFs is critical in maintaining good phase stability and in turn, the polarization of the shaped pulses. Using active phase stabilization, we can produce a phase stability of $\sim \lambda / 314$ between pulses from the two AOPDFs (Fig. 3a), which is, to our knowledge, the highest reported phase stability to date for pulse pairs generated by AOPDFs. In fact, it surpasses the

(a)

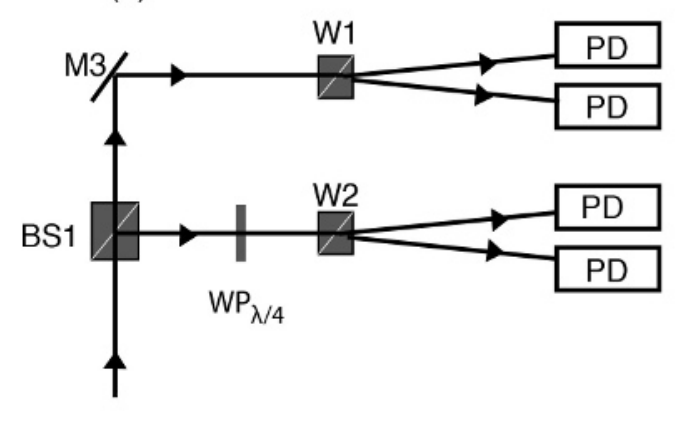

(b)

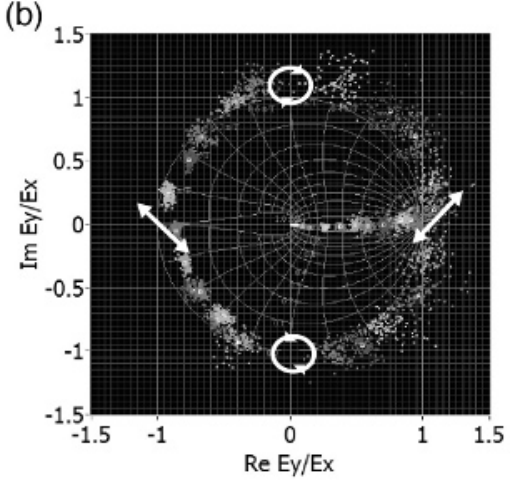

Fig. 2. (a) Polarization characterization is achieved by splitting the shaped pulse using a beam splitter (BS1) and subsequently Wollaston prisms (W1, W2) and measuring the intensity of the 
circularly and linearly polarized components using photo-diodes (PD). (b) Polarization measurements using the ellipsometer shown in (a). The data points are presented on a Poincaré sphere for easy visualization. Both linearly and circularly polarized pulses can be produced using the shapers shown in Fig.1a.

phase stability produced by a single AOPDF (Fig. 3b). In addition to unprecedented phase stabilization, this configuration also provides complete polarization control over each shaped pulse.

Fig. $2 \mathrm{~b}$ presents the polarization data on a Poincaré sphere for easy visualization. By controlling the spectral phase and amplitude of pulses from each AOPDF and combining them using PC2, we can prepare pulses in the desired polarization state. The data spanning the circumference of the Poincaré sphere correspond to polarization states of varying ellipticity, from right-handed to left handed circular polarization. The data along the equator correspond to linear polarization. This setup will be used to compare 2D spectra obtained using the two geometries shown in Fig. 1b, with the goal of exploring the advantages of phase-cycling and phase-matching techniques.
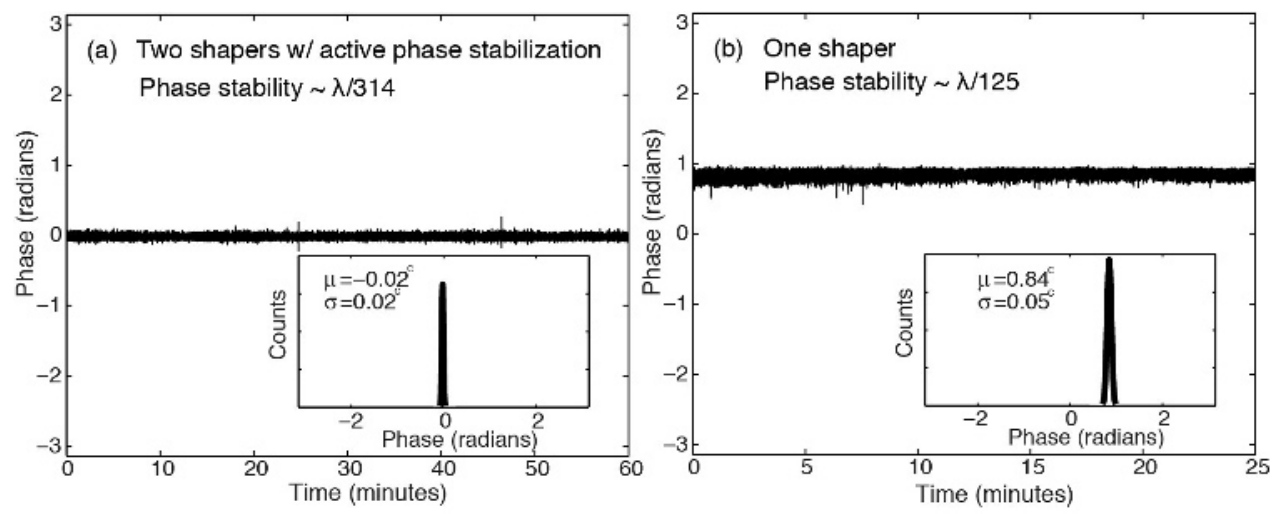

Fig. 3. Single-shot (1 kHz) phase measurements (using spectral interferometry) between two pulses (a) from two AOPDFs using active phase stabilization at $100 \mathrm{~Hz}$ over a period of 60 minutes, and (b) from a single AOPDF over a period of 25 minutes.

\section{References}

1. J. C. Vaughan, T. Hornung, K. W. Stone, K. A. Nelson, JPCA 111, 4873 (2007)

2. P. F. Tian, D. Keusters, Y. Suzaki, W. S. Warren, Science 300, 1553 (2003)

3. E. M. Grumstrup, S.H. Shim, M. A. Montgomery, N. H. Damrauer, M. T. Zanni, Opt. Exp. 15, 16681 (2007)

4. J. A. Myers, K. L. Lewis, P. F. Tekavec, J. P. Ogilvie, Opt. Exp. 16, 17420 (2008)

5. P.F. Tekavec, J. A. Myers, K. L. M. Lewis, and J. P. Ogilvie, Opt. Lett. 34, 1390 (2009) 